J. -P. Siedentopf M. Nagel

\title{
Substitutionstherapie und (psycho-)soziale Betreuung von opiatabhängigen Schwangeren. Aventis Early Morning Symposium, 13. Kongress der DGS, Berlin, 6.11.2004
}

Substitution Therapy and Psycho-(social) Counselling in Pregnant Opiate Addicts. Aventis Early Morning Symposium, 13th Conference of the DGS, Berlin, 6.11.2004

\section{Zusammenfassung}

Die Substitutionstherapie mit langwirkenden Opiaten oder Opioiden stellt die Standardtherapie für opiatabhängige Schwangere dar. Neben dem in Deutschland schon seit den 70er-Jahren genutzten Levomethadon (L-Polamidon ${ }^{\circledR}$ ) werden Methadon-Razemat und seit einigen Jahren auch Buprenorphin angewandt. Unter Beachtung einiger Grundregeln und in Kombination mit einer bereits präpartal einsetzenden psychosozialen Betreuung lassen sich weitgehend komplikationslose Schwangerschaftsverläufe erzielen. Der Artikel beleuchtet einige der wichtigsten Aspekte der Betreuung von opiatabhängigen Schwangeren.

\section{Schliuisselwörter}

Schwangerschaft · Substitution · Levomethadon · Methadon · Buprenorphin

\section{Abstract}

Substitution therapy with long-acting opiates and opioids is the state-of-the art treatment for pregnant opiate addicts. In the 1970 's levomethadone (Polamidon ${ }^{\circledR}$ ) was the only approved substance in Germany. In later years racemic methadone and buprenorphine were introduced as a treatment option. With adherence to some basic principles of opiate substitution therapy and in combination with intensive pre-partum psycho-social counselling only few risks are associated with these pregnancies. The article outlines some of the most important aspects for the treatment of pregnant opiate addicts.

Key words

Pregnancy $\cdot$ substitution therapy $\cdot$ levomethadone $\cdot$ methadone buprenorphine
Die Infektionsambulanz der Klinik für Geburtsmedizin am Campus Virchow-Klinikum der Charité - Universitätsmedizin Berlin - besteht seit 1987. Einen Schwerpunkt der Arbeit stellt die medizinische und psychosoziale Betreuung und Versorgung drogenabhängiger schwangerer Frauen dar. Jährlich stellen sich etwa 60 neue drogenabhängige und/oder substituierte Patientinnen in unserer Ambulanz vor, von denen ca. 45 bis zur Entbindung durch die Infektionsambulanz betreut werden. Neben der Betreuung schwangerer süchtiger Frauen ist ein wichtiger Teil unserer Arbeit die Aufklärung über Möglichkeiten der Familienplanung für süchtige Frauen. Bei Kinderwunsch sollen durch Vermeidung von riskanten Konsummustern möglichst gute Voraus- setzungen geschaffen werden, andererseits zeigt die hohe Zahl von Schwangerschaftsabbrüchen bei drogenabhängigen und substituierten Frauen, dass ein großes Defizit an Informationen über die Verhütung ungewollter Schwangerschaften besteht (Tab. 1).

Die Betreuung von süchtigen Schwangeren soll helfen, den Verlauf der Schwangerschaft so risikoarm wie möglich zu gestalten, die Patientin gesundheitlich und sozial weitgehend zu stabilisieren, sie in bestehende Hilfsangebote langfristig einzubinden und den Entzug des Kindes zu minimieren bzw. wenn möglich zu vermeiden. 
Tab. 1 Vergleich der anamnestisch angegebenen Schwangerschaftsabbrüche

\begin{tabular}{lll}
\hline $\begin{array}{l}\text { Anzahl der Schwanger- } \\
\text { schaftsabbrüche }\end{array}$ & $\begin{array}{l}\text { Perinatalstatistik } \\
\text { Berlin (alle Geburten } \\
\text { des Jahres 2001 in } \\
\text { Berlin) } \\
n=29.625\end{array}$ & $\begin{array}{l}\text { drogenabhängige } \\
\text { Schwangere der In- } \\
\text { fektionsambulanz } \\
1998-2002 \\
n=167\end{array}$ \\
\hline keiner & $71,3 \%$ & $92(54,4 \%)$ \\
\hline einer & $12,3 \%$ & $44(26 \%)$ \\
\hline zwei und mehr & $4,6 \%$ & $18(10,7 \%)$ \\
\hline drei und mehr & k. A. & $13(7,7 \%)$ \\
\hline
\end{tabular}

Für jede Patientin muss eine individuelle Behandlungsstrategie, die ein hierarchisches Vorgehen bezogen auf die suchtmedizinischen, psychosozialen, geburtshilflichen und anderen gesundheitlichen Anforderungen der Patientin berücksichtigt, entwickelt werden. Oft steht eine stabile, auf die Patientin abgestimmte Substitution zunächst im Vordergrund, gefolgt von der Regelung sozialer Grundbedürfnisse, die erfüllt werden müssen, damit eine regelmäßige und angemessene medizinische Versorgung und Betreuung der Patientin möglich wird.

Schwangere sind zumeist sehr motiviert, ihr bisheriges Leben und Konsumverhalten so zu verändern, dass ihr Kind keinen Schaden erleidet und die bestmöglichen Voraussetzungen hat. Dafür ist die Information über besonders riskante Konsummuster (Alkohol, Kokain) und Verhaltensweisen (Promiskuität) Voraussetzung.

Wichtigste Ziele bei der Betreuung von drogenabhängigen Schwangeren sind die Verhinderung von illegalem Drogenkonsum und die Vermeidung von Entzugssymptomen der Schwangeren, um Komplikationen wie vorzeitige Wehentätigkeit und Frühgeburtlichkeit zu verhindern [1]. Dies kann durch eine stabile Substitution mit langwirkenden Opioiden erreicht werden.

Als Substitutionsmedikamente in der Schwangerschaft bevorzugen wir L-Polamidon und Buprenorphin. L-Polamidon hat gegenüber Methadon-Razemat den Vorteil einer größeren pharmakologischen Sicherheit (Fertigarzneimittel, selten Auftreten von Long-QT-Syndrom oder Torsade-des-Points beschrieben) sowie eine geringere Substanzbelastung des Neugeborenen. Eine Einstellung der opiatabhängigen Schwangeren und die Umstellung von Methadon-Razemat auf L-Polamidon ist jederzeit möglich und unproblematisch.

Buprenorphin führt im Vergleich zu Methadon und L-Polamidon seltener zu einem Neugeborenen-Entzugssyndrom, das zudem in der Regel noch geringer ausgeprägt ist; es wird als Substitutionsmedikament jedoch nicht von allen Patientinnen akzeptiert $[2,3]$. In Abb. 1 wird die Häufigkeitsverteilung der angewandten Substitutionsmittel dargestellt.

Im Schwangerschaftsverlauf wird neben der regelmäßigen gynäkologischen Schwangerenvorsorge insbesondere eine psychosoziale Stabilisierung angestrebt. Durch eine interdisziplinäre

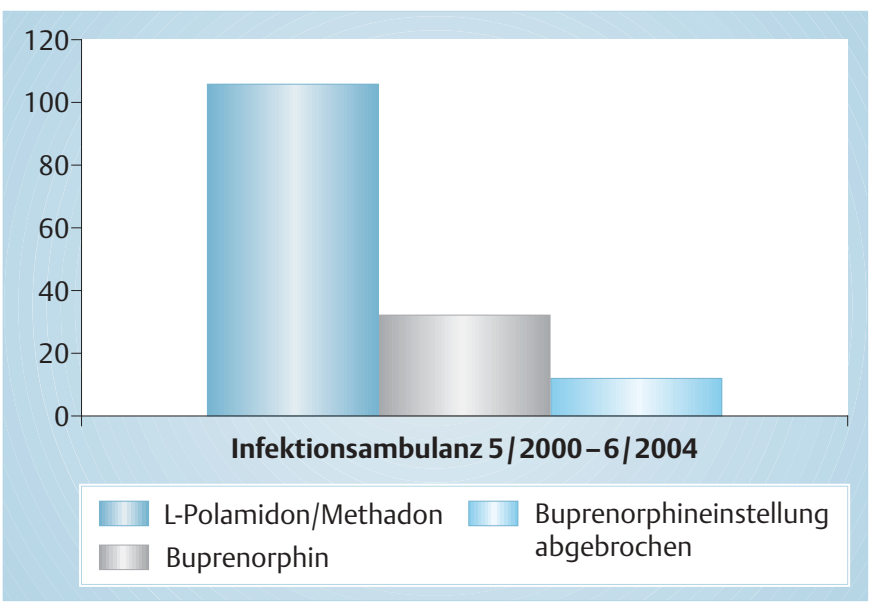

Abb. 1 Häufigkeitsverteilung der in der Infektionsambulanz angewandten Substitutionsmittel im Schwangerschaftsverlauf $(n=150)$.

Betreuung, zu der im Bedarfsfall z.B. auch Psychiater und Infektiologen hinzugezogen werden, können Risiken frühzeitig erfasst und behandelt werden. Aus suchtmedizinischer, geburtshilflicher und neonatologischer Sicht steht die beigebrauchsfreie Substitution im Vordergrund. Sofern dies von der Patientin angestrebt wird und es auch medizinisch vertretbar erscheint (keine Zunahme des Beigebrauchs!), kann mit einer langsamen Dosisreduktion (maximal 2,5 mg Polamidon/Methadon pro Woche) die Schwere und Häufigkeit eines Neugeborenen-Entzugssyndroms reduziert werden. In Einzelfällen kann während der Schwangerschaft der komplette Opiatentzug erreicht werden [3-6].

Die Entbindung einer drogenabhängigen Schwangeren sollte in einem Zentrum mit angeschlossener Neonatologie geplant werden, da insbesondere bei polyvalentem Substanzkonsum vor der Entbindung schwere akute Atemnotsyndrome der Neugeborenen auftreten können, die mitunter sogar eine Intubation erforderlich machen.

Die üblichen Substitutionsmedikamente sollten in jeder Klinik jederzeit verfügbar sein und der anamnestische und aktuelle Substanzkonsum der Patientin sowie der Status eventuell bestehender Begleiterkrankungen, insbesondere HIV und Hepatitis, sollten bekannt sein, damit Mutter und Kind optimal versorgt werden können.

Wenn die Mutter HIV-negativ ist, sollte die Empfehlung zum Stillen oder Abstillen nach individuellen suchtmedizinischen und psychosozialen Gesichtspunkten getroffen werden. Informationen zur Betreuung HIV-positiver Schwangerer finden Sie unter http://www.uni-duesseldorf.de/WWW/AWMF/ll/055-002.htm

Im Wochenbett kann sich der Bedarf bezüglich des Substitutionsmedikaments und der Tagesdosierung ändern. Um die Stabilität der Mutter nicht zu gefährden, muss dies angemessen berücksichtigt werden.

Ein stabiles soziales Umfeld vorausgesetzt, können etwa 80\% der Kinder unserer Patientinnen nach abgeschlossener Behandlung zur Mutter oder den Eltern entlassen werden. 
Für Fragen bezüglich der Substitution und Betreuung von opiatabhängigen Schwangeren oder Literaturanfragen stehen die Autoren gerne zur Verfügung.

\section{Literatur}

${ }^{1}$ Rementeria JL, Nunag NN. Narcotic withdrawal in pregnancy: stillbirth incidence with a case report. Am J Obstet Gynecol 1973; 116 (8): $1152-1156$

2 Fischer $\mathrm{G}$ et al.Treatment of opioid-dependent pregnant women with buprenorphine. Addiction 2000; 95 (2): 239-244
${ }^{3}$ Siedentopf JP et al. [Erfahrungen mit der Buprenorphineinstellung und anschliessenden Dosisreduktion im Vergleich zu L-Methadon bei schwangeren Opiatabhängigen]. Geburtsh Frauenheilk 2004; 64: $711-718$

${ }^{4}$ Kastner R et al. [Specific concepts of care for pregnant women with drug addiction or HIV infection]. Zentralbl Gynakol 2001; 123 (1): $48-50$

${ }^{5}$ Maas $\mathrm{U}$ et al. Infrequent neonatal opiate withdrawal following maternal methadone detoxification during pregnancy. J Perinat Med 1990; 18 (2): $111-118$

${ }^{6}$ Dashe JS et al. Opioid detoxification in pregnancy. Obstet Gynecol 199892 (5): $854-858$ 